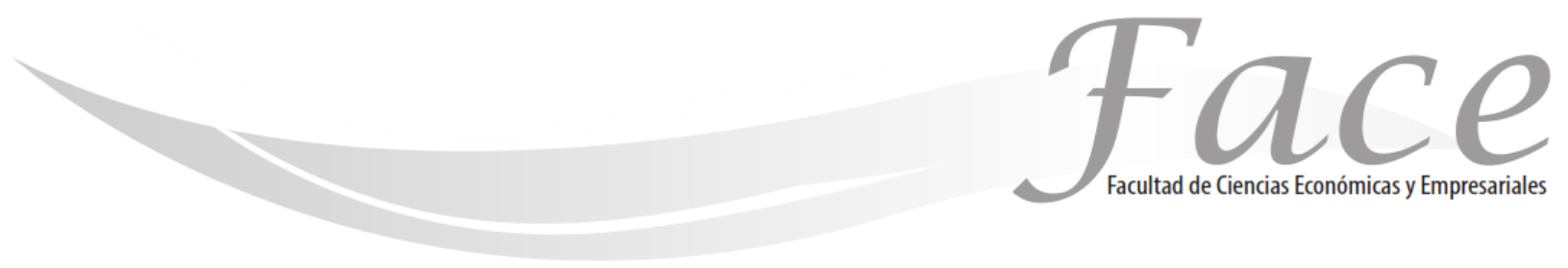

ISSN 1794-9920

Volumen $15-2$

Año 2015

Págs. 52 - 66

\title{
LA GENERACIÓN DE BENEFICIOS SOCIALES Y ECONÓMICOS DE LAS ZONAS FRANCAS EN COLOMBIA
}

\author{
Enrique Pinzón Alvarez * \\ Julio Enrique Lora Suarez **
}

Fecha de Recepción: 12 de Septiembre 2015

Fecha de Aprobación: 3 de Diciembre 2015

\section{Resumen:}

Este artículo analiza los costos desde el punto de vista económico y social para el estado, al desarrollar zonas francas en el territorio nacional y su impacto para las regiones, en la generación de empleo, ingresos, aumento de la producción, de valor e inversiones. Este documento se elaboró con información directa de cada una las zonas francas y las empresas ubicadas en esos territorios, tomando indicadores financieros, contables y de inversión. Con base en el modelo del Banco Interamericano de Desarrollo (BID) se desarrolló una matriz que arrojo los resultados del estudio.La metodología es de tipo explicativo, analiza los efectos y causas de un modelo que apoya la creación de empresas en zonas especiales, con beneficios tributarios y crecimiento productivo con destino al mercado nacional e internacional. El estudio concluye con la presentación de los impactos favorables y debilidades que para el estado y las empresas han tenido los beneficios económicos y sociales con la implantación del modelo de zonas francas.

Palabras Claves: Zonas francas especiales e uniempresariales, beneficios económicos y sociales, inversión, EVA®.

Clasificación JEL

JEL: F13, F14

\footnotetext{
* Magíster en Economía y Administración Financiera. Universidad Santo Tomás. Licenciado en Comercio Internacional. Universidad Jorge Tadeo Lozano Docente Universidad Tadeo Lozano - Colombia. Contacto: enrique.pinzon@utadeo.edu.co

** Magister Universidad de Los Andes - Colombia. Especialista en Finanzas y Evaluación de Proyectos - Colegio Mayor de Nuestra Señora del Rosario. Ingeniero Industrial de Pontificia Universidad Javeriana - Puj - Sede Bogotá. Colombia. Docente Universidad Tadeo Lozano - Colombia.

Contacto: julioe.lora@utadeo.edu.co
} 


\title{
THE GENERATION OF SOCIAL AND ECONOMIC BENEFITS IN COLOMBIA'S FREE TRADE ZONE
}

\begin{abstract}
:
This article analyzes the costs for the state from an economic and social perspective when developing free trade zones in the country and its impact on the regions such as job creation, income, and increase in production and investment value. Information was collected from each of the zones and businesses located in those areas, and were based on financial, accounting and investment indicators and an Inter-American Development Bank (BID) model. The research used an explanatory approach, analyzed the effects and causes of a model that supports the creation of companies in special zones, with tax benefit, productive growth and production destined for the national and international markets. Finally, the study presents both the positive and negative social and economic impact for the state and companies due to the implementation of a free trade zone model.
\end{abstract}

Keywords: Special Free Zones and Single-firms, Economic and Social Benefits, Investment, EVA®.

Classification JEL: F13, F14

\section{A GERAÇÃO DE BENEFÍCIOS SOCIAIS E ECONÓMICOS DAS ZONAS FRANCAS NA COLÔMBIA}

\section{Resumo:}

Este artigo analisa os custos desde o ponto de vista econômico e social para o estado, ao desenvolver zonas francas no território nacional e seu impacto para as regiões, em relação à geração do emprego, renda, aumento da produção, valor e investimento.

Este documento foi elaborado com informação direta de todas as zonas francas e empresas instaladas nesses territórios, utilizando indicadores financeiros, contábeis e de investimento. Com base no modelo do Banco Interamericano de Desenvolvimento (BID), desenvolveu-se uma matriz a qual revelou os resultados da pesquisa. A metodologia foi tipo explicativo, analisa os efeitos e as causas de um modelo que apoia à criação de empresas em zonas especiais, com benefícios tributários e crescimento produtivo destinado ao mercado nacional e internacional. 0 estudo conclui com a apresentação dos impactos favoráveis e as debilidades que tiveram os benefícios económicos e sociais para o estado e as empresas com a implantação do modelo de zonas de francas

PALAVRAS-CHAVE: Zonas Francas Especiais e Empresas Individuais, Benefícios Económicos e Sociais, Investimento, EVA®. 


\section{INTRODUCCIÓN:}

En Colombia se han incrementado las zonas francas como motores de desarrollo regional, transferencia de tecnología, y generadoras de industrias y servicios para el mercado internacional e interno. De manera más general, contribuyen al crecimiento de la economía y, por ende, se han convertido en creadoras de ingresos para el Estado al aportar recursos a través de los impuestos directos, como los de renta, e indirectos como el IVA y arancel de aduanas.

Este artículo permite cuantificar los beneficios económicos, mirando el comercio exterior, la producción, las inversiones recibidas por las empresas ubicadas en las zonas francas y los beneficios sociales, como empleo, aportes a salud, pensiones y cajas de compensación (Hoy- CREE'1), también se buscó ponderar si realmente las zonas francas han contribuido al desarrollo del sector manufacturero, las exportaciones, la generación de impuestos, montos de inversión y en qué sectores se especializa cada región.
De otro lado permite identificar, según fuente DIAN, la existencia de 578 empresas ubicadas en zonas francas, hasta abril 2013, de las cuales se obtuvo información de 509 empresas; 293 de servicios y 216 industriales. En servicios sobresalen comercializadoras con 85 sociedades, logística 50 , almacenamiento 28 , telecomunicaciones 28 y otras 102. En las industriales se destacan alimentos y bebidas con 28 establecimientos, confecciones 25, metalmecánica 24 , plástico y caucho 21 , maquinaria y equipos electrónicos 19 y otros 99. El decreto 4051 de 2007 , establece que solo puede existir $5 \%$ de empresas comerciales, pero las razones sociales de los usuarios en zonas francas incluyen actividades de industria combinadas con comercio, evadiendo la referida condición.

\section{METODOLOGÍA:}

De tipo explicativo, busca analizar los efectos y causas de un modelo que apoya la creación de empresas en zonas éspeciales, con beneficios tributarios y crecimiento prciductivo con destino al mercado nacional e internacional. Indicadores zonas francas según modelo.

\begin{tabular}{|c|c|c|c|c|c|c|c|}
\hline INDICADOR & 2006 & 2007 & 2008 & 2009 & 2010 & 2011 & 2012 \\
\hline PRODUCCION & $21,878,562$ & $25,841,332$ & $80,548,200$ & $55,372,182$ & $97,187,462$ & $112,300,361$ & $86,033,639$ \\
\hline $\begin{array}{l}\text { UTILIDAD ANTES DE } \\
\text { IMPUE STOS }\end{array}$ & $(35,581)$ & $(5,320)$ & 622,047 & 516,050 & 454,169 & 749,795 & 892,770 \\
\hline IMPUE STO RENTA PAGADO & 1,264 & 3,834 & 200,731 & 213,333 & 239,958 & 298,211 & 353,781 \\
\hline IMPORTACIONES US & 123,658 & 632,114 & $1,778,824,156$ & $1,506,808,953$ & $2,510,128,724$ & $3,295,821,882$ & $4,060,874,439$ \\
\hline EXPORTACIONES US & - & - & $247,517,762$ & $199,615,364$ & $1,395,869,705$ & $2,280,233,257$ & $2,310,165,561$ \\
\hline IMPUE STO ARANCEL & 10,511 & 53,730 & $151,200,053$ & $128,078,761$ & $213,360,942$ & $280,144,860$ & $345,174,327$ \\
\hline VENTAS TOTALES & 313,971 & 411,475 & $17,048,829$ & $15,994,657$ & $17,983,937$ & $24,121,245$ & $100,879,785$ \\
\hline IMPUE STO IVA & 50,235 & 65,836 & $2,727,813$ & $2,559,145$ & $2,877,430$ & $3,859,399$ & $16,140,766$ \\
\hline INVERSION & $10,237,780$ & $12,982,477$ & $27,971,107$ & $9,820,832$ & $29,027,013$ & $33,455,398$ & $14,330,074$ \\
\hline UTILIDAD OPERACIONAL & - & 40,328 & 941,083 & 630,416 & 619,201 & $1,140,691$ & $1,012,021$ \\
\hline CAPITAL INVERTIDO & $23,754,441$ & $26,905,115$ & $48,848,407$ & $14,941,149$ & $49,198,388$ & $57,969,955$ & $22,751,633$ \\
\hline UTILIDAD NETA & $(38,395)$ & $(2,435)$ & 430,991 & 299,470 & 243,658 & 505,391 & 556,381 \\
\hline $\begin{array}{l}\text { SALARIO +CARGA } \\
\text { PRE STACIONALY } \\
\text { PARAFICALES }\end{array}$ & $1,568,070,000$ & $3,581,809,000$ & $\begin{array}{c}419,136,858,00 \\
0\end{array}$ & $\begin{array}{c}620,444,034,00 \\
0\end{array}$ & $\begin{array}{c}649,841,220,00 \\
0 \\
\end{array}$ & $\begin{array}{c}706,869,450,00 \\
0\end{array}$ & $\begin{array}{c}706,869,450,00 \\
0\end{array}$ \\
\hline ACTIVOS FIJOS & $42,314,214$ & $45,210,141$ & $65,107,394$ & $16,176,716$ & $75,293,040$ & $85,721,003$ & $33,661,915$ \\
\hline $\begin{array}{l}\text { IMPUESTO DE RENTA } \\
\text { EXONERADO }\end{array}$ & $(6,405)$ & (958) & 111,968 & 92,889 & 81,750 & 134,963 & 160,699 \\
\hline $\begin{array}{c}\text { PARAFICALES SENA-ICBF- } \\
\text { CAJAS }\end{array}$ & $11,411,685$ & $26,066,691$ & $3,050,283,339$ & $4,515,303,447$ & $4,729,242,510$ & $5,144,267,475$ & $5,144,267,475$ \\
\hline CREE & $(3,202)$ & (479) & 55,984 & 46,445 & 40,875 & 67,482 & 80,349 \\
\hline $\begin{array}{l}\text { VALOR ECONOMMICO } \\
\text { AGREGADO EVA }\end{array}$ & $(6,242,354)$ & $(6,754,245)$ & $(12,095,398)$ & $(4,617,411)$ & $(11,465,777)$ & $(13,578,079)$ & $(6,023,585)$ \\
\hline
\end{tabular}

${ }^{1}$ Aporte de las sociedades y personas jurídicas y asimiladas en beneficio de los trabajadores, la generación de empleo y la inversión social en los términos de la ley 1607 DE 2012. 


\section{LA GENERACIÓN DE BENEFICIOS SOCIALES Y ECONÓMICOS DE LAS ZONAS FRANCAS EN COLOMBIA}

Enrique Pinzón Álvarez - Julio Enrique Lora Suarez

Con base a la información suministrada por la DIAN sobre las 106 Zonas francas y las 706 usuarios al mes de abril de 2013, a través de la base datos Benchmark de la Universidad Jorge Tadeo Lozano, se obtuvo de cada empresa el balance de los últimos siete años (2006-2012), los indicadores económicos, impuestos de renta y parafiscales, generación de empleo, capital, activos, inversiones y comercio exterior, entre otros. Los datos se tomaron en valores, en millones de pesos y las exportaciones e importaciones en dólares americanos, en términos corriente.

La matriz cuenta con los principales indicadores, como producción, número de empleo, impuestos de renta, para fiscales (Sena, cajas de compensación familiar Instituto Colombiano de Bienestar familiar, hoy Cree), impuesto de valor agregado IVA, exportaciones, importaciones, inversión, entre otros. (Cuadro $N^{\circ} 1$ ).

Se clasificaron las empresas por dos grandes grupos, uno de servicio y el otro industrial. Además, se subdividieron por subsectores.(Ver grafica $\mathrm{N}^{\circ} 1$ y $\mathrm{N}^{\circ} 2$ )

Una vez obtenida la información se procedió a elabora una tabla dinámica en Excel en donde se determinaron los indicadores que se elaboraron para poder determinar los resultados del estudio, según fórmulas que se introdujeron para obtener la información esperada.

\section{MARCO TEÓRICO:}

El marco teórico de las zonas francas se puede enmarcar en los conceptos de las ventajas competitivas de Michael Porter, la teoría de los costos de los factores de Heckscher y Bertil Ohlin así como las ventajas comparativas de David Ricardo principalmente. Conceptos que retomaremos a continuación aplicándolos a este mecanismo que es considerado como un instrumento de apoyo a las exportaciones a nivel mundial. Partiendo del concepto de Ricardo de las ventajas comparativas en donde "un país exporta bienes en los cuales tiene un costo relativo ventajoso no un costo absoluto en comparación con otros países" (Torres, 2008).

Con esa definición, las zonas francas reducen sustancialmente, los costos al tener exención de impuestos arancelarios, eliminación o rebaja en los costos de transporte, disminución en el impuesto de renta, tarifas reducidas de los servicios y la exclusión de impuestos adicionales como los de industria y comercio y el impuesto de valor agregado, dando unas ventajas frente a industrias similares instaladas en el territorio nacional que tienen que cancelar todos los impuestos directos e indirectos.

Además, se puede complementar el concepto anterior con la abundancia de tierra y mano de obra, con la teoría de los costos de los factores de Heckscher y Bertil Ohlin, que generalmente es donde se ubican esos establecimientos; existen unos factores muy baratos y en algunos casos calificados, que pueden contribuir a disminuir los precios de los bienes obtenidos en dichos territorios y generar una mayor eficiencia en la producción, vía inversión extranjera y nuevas tecnologías.

Si se toma la teoría de la ventaja competitiva de Michael Porter, vemos que el diamante tiene una aplicación directa a las zonas francas; por una parte, pueden existir dentro de la zona industrial conexas a la producción o proveedoras de materias primas e insumos indispensables, igualmente, existen facilidades para traer sin costos de impuestos arancelarios del exterior los componentes de los bienes finales 0 productos intermedios y además, existe apoyo para los insumos baratos y se ahorran costos por la contratación de los servicios en grupo de las empresas ubicadas en el territorio delimitado por la zona.

\section{Las Zonas Francas en Colombia}

Como se mencionó, la primera zona franca en Colombia fue la de Barranquilla, creada en (1958), que sirvió para desarrollar una serie de industrias y servicios. Las otras seis creadas antes de la Ley 7 de 1991, fueron constituidas en la década del setenta; Palmira (1970), Cúcuta (1972), dos en Cartagena (1973), Santa Marta (1974) y la desaparecida zona franca oficial de Buenaventura (1972). Antes del 2004; además de las anteriores en los noventa se instituyeron la del pacifico (1992), Rio Negro (1993), Bogotá (1993), Armenia (1996) y Sopo en el (2000).

Como se puede observar no existen empresas de alta tecnología, en su mayoría son firmas de baja tecnología. (Gráfica No. 2).

El auge de estos establecimientos se dispararon entre los años 2008 y 2010, en especial las uniempresariales 0 especiales, con el fin de amparar las importaciones de materias primas e insumos, con tramites ágiles y sin pago del impuesto del arancel e IVA y para obtener la disminución del impuesto de renta, entre otros. 
Gráfica No. 1.

Número de empresas por sectores - Servicios

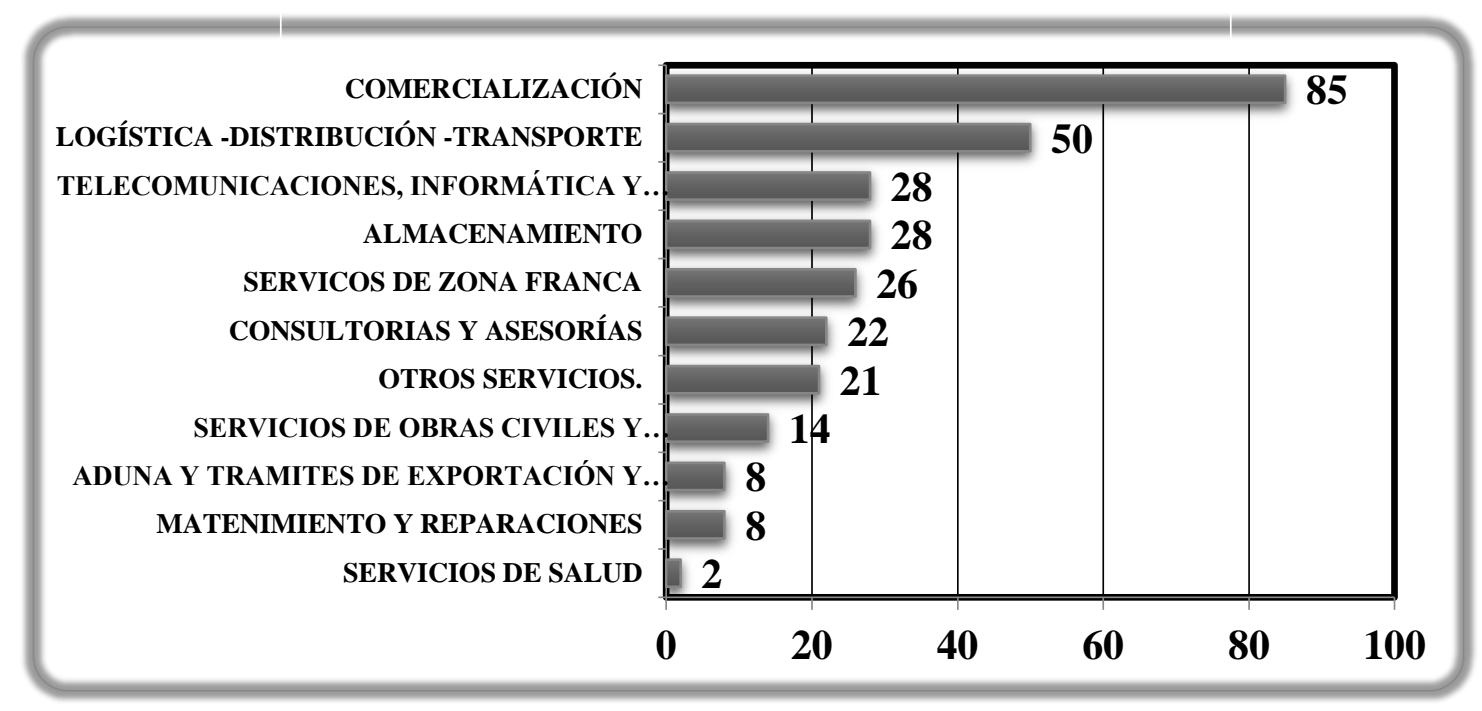

Fuente : Elaboración propia

De las empresas instaladas en las zonas francas evaluadas, el $58 \%$ de las empresas son de servicios (Gráfico No.1), entre las que se destacan las comercializadoras con (85) establecimientos, (50) de logística, almacenamiento, telecomunicaciones, informática y sistemas, cada una con (28) compañías y (26) que son zonas francas propiamente. (No se encontró información de 9 zonas francas de servicios, por cuanto están en construcción o no están operando todavía bajo esta modalidad).

El número de empresas instaladas del sector industrial asciende a 215 compañías distribuidas en alimentos y bebidas (28), confecciones textiles (25), metalmecánica (24), plásticos y caucho (21), máquinas y equipos electrónicos (19), farmacéuticos (17), químicos (14), también existen empresas de cuero y sus manufacturas, vidrio, petroquímicos, agroindustrial, cerámicas, artes gráficas, autopartes, entre otras. De los 32 departamentos en Colombia, 20 tienen en su territorio éstos establecimientos, concentrándose en Cundinamarca y Bolívar, cada uno cuenta con 14 zonas francas, seguidos por Atlántico, Antioquia, Cauca, y Valle del Cauca con 8, respectivamente, en aras de generar industrias, desarrollo tecnológico y empresas para desarrollar las regiones respectivas.

\section{Aportes Sociales}

En Colombia no existe un estudio que determine el impacto económico y social de las zonas francas, si bien el análisis cuantitativo solamente se efectúa para 7 años; lo ideal es que la serie fuera como mínimo de 10 años, pero es necesario tener en cuenta que la reforma se realizó en el 2005 con la ley 1004 y las solicitudes tanto de nuevas zonas franca como las uniempresalariales se presentaron en gran escala a partir del 2008 (Cuadro $N^{\circ} 2$ ). Para cuantificar los efectos sociales, además del empleo se miraron los aportes parafiscales, que fueron sustituidos por el impuesto de renta para la equidad Cree en el 2013, que entró a regir a partir del 2014.

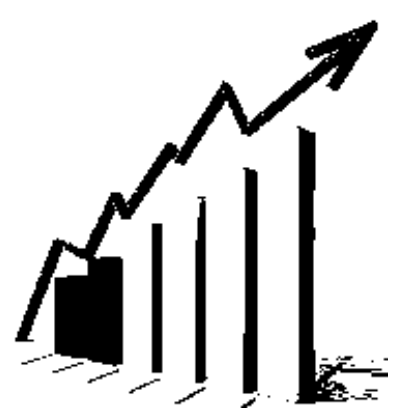


Cuadro $\mathrm{N}^{\circ} 2$.

Beneficios Sociales

\begin{tabular}{|c|c|c|c|c|c|c|c|}
\hline \multicolumn{7}{|c|}{ Valores \$ millones } \\
\hline INDICADOR & 2006 & 2007 & 2008 & 2009 & 2010 & 2011 & 2012 \\
\hline SALARIO & $1,026,000,000$ & $2,343,600,000$ & $274,244,400,000$ & $405,961,200,000$ & $425,196,000,000$ & $462,510,000,000$ & $462,510,000,000$ \\
\hline SALUD & $87,210,000$ & $199,206,000$ & $23,310,774,000$ & $34,506,702,000$ & $36,141,660,000$ & $39,313,350,000$ & $39,313,350,000$ \\
\hline PENSION & $123,120,000$ & $281,232,000$ & $32,909,328,000$ & $48,715,344,000$ & $51,023,520,000$ & $55,501,200,000$ & $55,501,200,000$ \\
\hline $\begin{array}{c}\text { RIESGO } \\
\text { PROFE SIONALES }\end{array}$ & $15,390,000$ & $35,154,000$ & $4,113,666,000$ & $6,089,418,000$ & $6,377,940,000$ & $6,937,650,000$ & $6,937,650,000$ \\
\hline $\begin{array}{c}\text { PARAFICALES SENA } \\
\text { ICBF CAJAS COM }\end{array}$ & $92,340,000$ & $210,924,000$ & $24,681,996,000$ & $36,536,508,000$ & $38,267,640,000$ & $41,625,900,000$ & $41,625,900,000$ \\
\hline $\begin{array}{c}\text { BENEFICIO TOTAL } \\
\text { BEA }\end{array}$ & $1,344,060,000$ & $3,070,116,000$ & $359,260,164,000$ & $531,809,172,000$ & $557,006,762,010$ & $605,888,100,000$ & $605,888,100,000$ \\
\hline No. EMPLEADOS & 95 & 217 & 25,393 & 37,589 & 39,370 & 42,825 & 42,825 \\
\hline
\end{tabular}

\section{Empleo}

Desde el 2006 al 2012 las empresas de zonas francas han generado 42.825 empleos directos, cumpliendo con el $80 \%$ de los compromisos en este tema, si tomamos el máximo de 500 puesto de trabajo por las 106 zona francas existentes, estipulados en el decreto 4051 de 2007 y demás normas reglamentarias de la ley 1004 de 2005. Las razones son obvias si tenemos en cuenta que mientras se aprueba una zona franca y se hacen las adecuaciones necesarias y la instalación de usuarios pueden pasar tres años 0 más. Es bueno aclarar que el promedio por empresa supera los 75 empleos, resultado que se debe fundamentalmente a las grandes empresas que se acogieron al mecanismo mencionado y ya de hecho existan puestos de trabajo.

Los años 2008, 2009 y 2012, presentan el mayor número de empleos, probablemente como resultado de la generación de estos establecimientos a partir de la reforma consagrada en el 2005. Por otro lado se puede apreciar que entre el 2011 y el 2012 no se ve un incremento del empleo por cuanto en el 2012, algunas empresas uniempresariales desistieron del mecanismo. (Siemens y Paz del Rio). Del total de las personas ocupadas en el país, 21.471.000 empleos en el 2012, según datos suministrados por el diario el tiempo del día 16 de enero del 2014 , las zonas francas contribuyeron con el $0.20 \%$.
Los sectores que más empleos generan, son el de textiles y confecciones $12.71 \% \quad(12.452)$, empresas de comercialización $\quad 11.98 \% \quad(11.736)$, productos farmacéuticos y cosméticos $\quad 9.29 \% \quad$ (9.098), almacenamiento $9.17 \%$ (8.982). Esto significa que las empresas que más genera empleo son de tecnología baja y mano de obra no muy calificada, con excepción de los medicamentos y farmacéuticos. (Grafica $\mathrm{N}^{\circ} 3$ ). Los sectores que menos empleo generan son los agroindustriales, servicios de salud y vidrio, de alta tecnología.

\section{Seguridad social}

El empleo a partir del 2008 se dispara tanto el número de empresas en las zonas francas, como en la generación de empleo, al pasar de 95 empleos en el 2006 a 25.393 en el 2008 incrementándose al igual los aporte prestacionales de Sena, Cajas de Compensación Familiar, Instituto Colombiano de Bienestar familiar, que pasaron de $\$ 92.340$ millones en el 2006 a \$ 41.625.900.000 millones en el 2012. Es a partir del 2008 en donde se generaron en forma creciente los recursos por los conceptos antes mencionados. El aporte de las empresas en salud hasta el 2012 era del $8.5 \%$, de la nómina, alcanzando los $\$ 39.313 .350 .000$ millones. El aporte en pensiones se calculó tomando el valor de la nómina por el $12 \%$, reflejando un valor de $\$ 55.501 .200 .000$ millones en el 2012. 
Salario

En cuanto a los riesgos profesionales se tomó la base de $1.5 \%$ del aporte y esta se calculó al igual que la anteriores, según el valor de la nómina, ascendiendo a $\$ 6.937 .650 .000$ millones. Lo que indica, si sumamos los aportes parafiscales, la salud, pensiones y riesgos profesionales, el valor generado por seguridad social de las zonas francas en el 2012 alcanzaron los \$ 143.378 .100 millones, cifra importante para un país como Colombia y representando un aporte social per cápita mes de $\$ 279.000$.
Para efectos de calcular los ingresos generados, se tomó como base un promedio de salario mensual de $\$ 900.000$ (Según encuesta de hogares Dane 2012) y lo multiplicamos por el número de empleados, el valor de la nómina mensual en el 2012 es de \$ 462.510.000.000 millones, que contribuyen al bienestar de los colombianos y a la generación de la demanda por bienes y servicios. Si al valor per cápita de los salarios le sumamos el aporte social unitario nos daría un valor de $\$ 1.179 .000$, cifra que ayuda aliviar uno de los problemas del país, cual es el de la desigualdad en los ingresos.

Gráfica No.3.

Generación de empleo zona franca

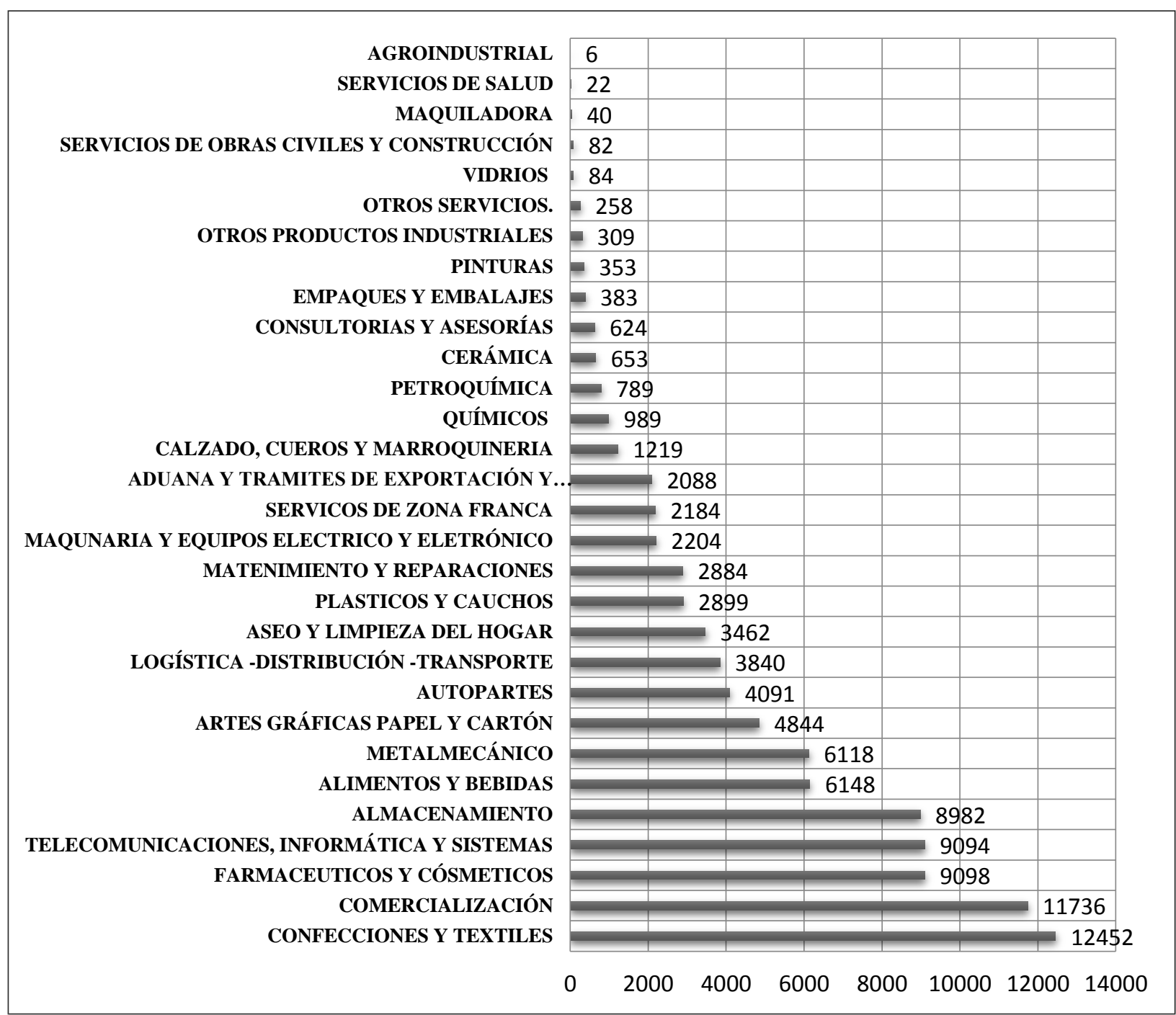

Fuente : Elaboración propia datos base de datos Benchmark 
Enrique Pinzón Álvarez - Julio Enrique Lora Suarez

son mayores los valores en las empresas de servicios que los industriales, en donde ya para el 2012 los valores de

Cuadro No. 3

Beneficios económicos.

\begin{tabular}{|c|c|c|c|c|c|c|c|}
\hline \multicolumn{8}{|c|}{ Valores en \$ Millones } \\
\hline INDICADOR & 2006 & 2007 & 2008 & 2009 & 2010 & 2011 & 2012 \\
\hline $\begin{array}{l}\text { PRODUCCION } \\
\text { INDUSTRIAL }\end{array}$ & 128,076 & 152,654 & $40,559,238$ & $41,219,005$ & $43,654,732$ & $50,846,841$ & $55,562,580$ \\
\hline $\begin{array}{l}\text { PRODUCCION } \\
\text { SERVICIOS }\end{array}$ & $21,750,486$ & $25,688,678$ & $39,988,962$ & $14,153,177$ & $53,532,730$ & $61,453,520$ & $30,471,059$ \\
\hline $\begin{array}{l}\text { IMPUESTOS IVA } \\
\text { CANCELADO }\end{array}$ & 38,681 & $5,069,372$ & $2,100,416$ & $1,970,542$ & $2,215,621$ & $2,971,737$ & $12,428,390$ \\
\hline IMPUESTOS TOTAL IVA & 50,235 & 65,836 & $2,727,813$ & $2,559,145$ & $2,877,430$ & $3,859,399$ & $16,140,766$ \\
\hline INVERSION & $10,237,780$ & $\begin{array}{c}129,982,47 \\
7\end{array}$ & $27,971,107$ & $9,820,832$ & $29,027,013$ & $33,455,398$ & $14,330,074$ \\
\hline TOTAL PATRIMONIO & $77,090,528$ & $86,245,594$ & $132,993,696$ & $45,770,911$ & $146,344,612$ & $177,321,176$ & $83,622,155$ \\
\hline UTILIDAD NETA & $(38,395)$ & $(2,435)$ & 111,968 & 92,889 & 81,750 & 134,964 & 556,381 \\
\hline $\begin{array}{c}\text { IMPUESTOS RENTA } \\
\text { PAGADOS }\end{array}$ & 353,781 & 353,781 & 353,781 & 353,781 & 353,781 & 353,781 & 353,781 \\
\hline IMPORTACIONES US\$ & 123,658 & 632,114 & $1,778,824,156$ & $1,506,808,953$ & $2,510,128,724$ & $3,295,821,882$ & $4,060,874,439$ \\
\hline EXPORTACIONES US\$ & - & - & $247,517,762$ & $199,615,364$ & $1,395,869,705$ & $2,280,233,257$ & $2,310,165,561$ \\
\hline $\begin{array}{l}\text { BALANZA ZONAS } \\
\text { FRANCAS US\$ }\end{array}$ & $(123,658)$ & $(632,114)$ & $\begin{array}{c}(1,531,306,39 \\
4)\end{array}$ & $\begin{array}{c}(1,307,193,58 \\
9)\end{array}$ & $\begin{array}{c}(1,114,259,01 \\
9)\end{array}$ & $\begin{array}{c}(1,015,588,62 \\
5)\end{array}$ & $\begin{array}{c}(1,750,708,87 \\
8)\end{array}$ \\
\hline BALANZA EN PESOS & (279) & 1,273 & $(3,449,604)$ & $(2,636,674)$ & $(2,145,906)$ & $(1,964,229)$ & $(3,140,667)$ \\
\hline TAL BENEFICIO & $109,258,655$ & $247,206,010$ & $246,653,931$ & $115,799,834$ & $277,973,846$ & $330,341,246$ & $213,272,104$ \\
\hline
\end{tabular}

Fuente: Elaboración propia

\section{Aportes Económicos}

Par determinar el aporte económico de estos establecimientos debemos mirar la producción, las exportaciones, las importaciones, las inversiones, los impuestos de renta, Impuestos del valor agregado, inversiones y patrimonio. Sin embargo, al considerar el aporte económico no incluimos los valores sociales (Cuadro N³).

\section{Producción}

La producción en los siete años del estudio se ha triplicado al pasar de $\$ 21.8$ billones en el 2006 a $\$ 86,03$ billones en el 2012. Como cosa curiosa entre los años 2006 al 2011 producción industrial superan a los servicios en más de $\$$ 25,09 billones. Es mayor el número de empresas prestadora de servicio, aunque existe en la norma una reglamentación para tener un mínimo empresas de servicios, pero si se mira los objetivos actuales de dichos establecimientos se incluye el término de empresas de producción, transformación y de servicios. Los subsectores que más aportan a la actividad productiva son Metalmecanica con el $31.8 \%$, alimentos y bebidas $17,4 \%$, confecciones y textile $17,09 \%$, petroquimica $16.5 \%$, artes graficas, papeles y cartone $8,2 \%$. 


\section{Comercio Exterior}

Para poder visualizar mejor el comercio exterior de las zonas francas tenemos que mirar el comportamiento de los últimos seis años bajo los datos que procesa la Dian y la información suministrada por las empresas.(Cuadro No.4).

\section{Cuadro $\mathrm{N}^{\circ} 4$}

Exportaciones zonas francas.

\begin{tabular}{|c|c|c|c|}
\hline \multicolumn{4}{|c|}{ EXPORTACIONES TOTALES ZONAS FRANCAS } \\
\hline \multicolumn{4}{|c|}{ FOB MILES DE DOLARES US } \\
\hline AÑO & PAIS & ZONAS FRANCAS & ZONAS ESPECIALES \\
\hline & VALORES US & PARTICIPACIÓN \% & PARTICIPACIÓN \% \\
\hline 2009 & 32.852 .994 & $2.19 \%$ & $0.20 \%$ \\
\hline & & & $3.56 \%$ \\
\hline 2010 & 39.819 .528 & $5.14 \%$ & $4.29 \%$ \\
\hline & & & $5.60 \%$ \\
\hline 2011 & 56.9563 .500 &
\end{tabular}

Fuente: Elaboración propia con datos de DANE

Existe un pequeño crecimiento como se puede observar en el (Cuadro No.4), en donde la participación dentro de las exportaciones totales representaron en el año 2009 tan solo el 2,19\% y en el 2011 el 5,4\%, lo que supone que estos establecimientos son más para el mercado nacional que para los mercados internacionales. La balanza comercial de las zonas francas desde el año 2006 ha sido negativa. El saldo negativo mayor fue el del 2012 que alcanzó los US\$1.750.7 millones. Como resultado neto de los ingresos menos los egresos a las zonas francas, al país le representó en el 2011, un beneficio de tan solo US\$ 975 millones, que no se compadecen desde el punto de vista comercial, por ahora, con las grandes inversiones en dichos establecimientos. Se debe mirar con mucho cuidado la generación de empleo, la demanda agregada que se supone debe crear, la incidencia en el área de influencia, la infraestructura y los servicios.

La Balanza comercial, el comercio exterior de las zonas francas son negativos en US\$ 1.750 .7 millones en el 2012, aunque a partir del 2011 la tendencia era a la disminución, el último año se disparo, pero lo intersante es ver como las exportaciones a partir del 2008 se incrementaron en forma continua al pasarde US\$ 247 millones a US $\$ 2.310$ milones en el 2012; dato si se mantiene, indicaría que muy pronto estos estabecimientos van ha generara superavit en la balanza comercial.

\section{Inversión}

Los datos de inversión fueron tomados directamene del patrimonio de la compañía, que reflejan verdaderamente el valor dedicado a cada actividad. El mayor monto de inversión se da en el año 2011 en donde alcanzó los \$177.321.176 millones y comienza a descender en el 2012

a los niveles del 2007. Tambien, se puede ver como la crisis económica mundial afecto la inversión en zonas francas por la vertiginosa caida en el 2008 al 2009. Si confirma lo ya dicho anteriormente que en los últimos cuatro años fueron los de mayor número de establecimientos y creación de zonas francas. Según datos del Ministerio de Comercio Industria y Turismo los mayores montos de inversión en las 106 zonas francas efectuados hasta abril del 2013, ascendiero a \$ 7.538 millones de los cuales en los departamentos de Bolivar invirtieron \$ $\$ 3.681 .5$ millones $(50 \%)$ y Boyacá con $\$$ 2.264 .5 millones $(30.7 \%)$, si bien en 20 departamenteo existen zonas franza, los montos de inversión no son tan significativas en los demás departamentos, como los dos anteriores.

\section{Impuestos}

Los impuestos que cancelan las empresas ubicadas en las áreas delimitadas son el de renta, arancel, IVA cuando venden al mercado nacional y los para fiscales (hoy CREE). Los impuestos de renta pagados versus los exonerados presentan una diferencia progresiva de $\$ 193.082$ millones más en el pago. Las razones son fundamentalmente el desmonte de la exoneración y el pago del $15 \%$ sobre la utilidad obtenida apartir de la ley 1004 del 2005 ( Gráfica No. 4).

\section{Impuesto IVA}

Es claro que la entrada de productos a zonas francas no pagan el impuesto de valor agregado, sin embargo,se debe tener en cuenta que al ingresar al mercado nacional los bienes obtenidos dentro de ellas se les consideran como una importación y deben para efectos de su nacionalización pagar el referido impuesto sobre el insumo proveniente de terceros países.

El ingreso por este concepto paso en el 2006 de $\$ 38.681$ millones a $\$ 12.428 .390$ millones, en el 2012, esto es un $321 \%$ de crecimiento, lo que equivale a un promedio en los siete años de $46 \%$, bastante significativo para las arcas del estado. El iva exonerado pasó de \$ 11.554 millones en el 2006 a \$3.712.396 millones en el 2012, comportamiento similar al pagado. Representado el $30 \%$ del valor recaudado en promedio, correspondiante a los productos enviados al exterior. 
Gráfica No. 4.

Impuesto de renta

\begin{tabular}{|c|c|c|c|c|c|c|c|c|}
\hline \multirow{9}{*}{ 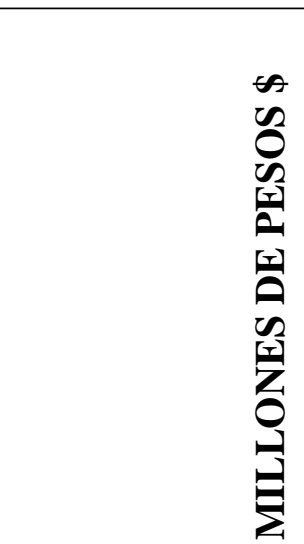 } & \multirow{2}{*}{$\begin{array}{l}400000 \\
350000\end{array}$} & & & & & & & \\
\hline & & & & & & & & \\
\hline & 300000 & & & & & & & \\
\hline & 250000 & & & & & & & \\
\hline & 200000 & & & & & & & \\
\hline & 150000 & & & & & & & $\ldots$ \\
\hline & 100000 & & & & & & & \\
\hline & 50000 & & & & & & & \\
\hline & 0 & $\Delta$ & & & & & & \\
\hline & -50000 & 1 & 2 & 3 & 4 & 5 & 6 & 7 \\
\hline AÑOS & & 2006 & 2007 & 2008 & 2009 & 2010 & 2011 & 2012 \\
\hline $\begin{array}{r}--\boldsymbol{-}-\text { IMPUESTO DE R } \\
\text { EXONERAD }\end{array}$ & $\begin{array}{l}\text { ENTA } \\
\mathrm{O}\end{array}$ & (6.405) & (958) & 111.968 & 92.889 & 81.750 & 134.963 & 160.699 \\
\hline 一_- IMPUESTO REN & TA PAGADO & 1.264 & 3.834 & 200.731 & 213.333 & 239.958 & 298.211 & 353.781 \\
\hline
\end{tabular}

Fuente: Elaboración propia con datos de DANE

\section{Costos de las zonas francas}

Los costos para el estado se reflejan en los montos exonerados en los impuestos de renta, el impuesto de valor agregado IVA, el arancel dejado de cancelar y el déficit de la balanza comercial , en estos datos no se calculó el valor de los gastos del estado en infraestructura porque no fue posible su consecución (Cuadro $\mathrm{N}^{\circ} 5$ ).

Los mayores costos del estado son los impuestos del arancel, que como se puede apreciar pasaron en menos de 7 años de $\$ 10.511$ millones en el 2006 a $\$ 345.174 .327$ millones en el 2012, representando el $98 \%$ de los cotos totales, como resultado del auge de empresa instaladas en zonas francas, en los tres último años.

Con respecto al impuesto de renta, si bien el esquema se cambió, hoy los usuarios tienen que pagar el 15\%,( no aplica a los comerciales) y no como antes que tenían una exención total, por eso se ve un incremento gradual del $335 \%$ promedio anual desde el 2008 . El déficit de la balanza comercial es progresivo desde el año 2008, hasta alcanzar las suma de \$352.188.069 millones en el 2012. Además, con los datos anteriores se confirma que las zonas francas en Colombia son más para el mercado nacional que para atender mercados externos.
Si miramos los costos para el estado, frente a los beneficios económicos obtenidos, en los años 2008 y 2012, representaron un $63 \%$ y $165 \%$ respectivamente, esto significa que para generar un peso en zonas francas se necesitan \$1.65, en el 2012. Para los años 2006 al 2011 se situaron en promedio en el $80 \%$, siendo relativamente elevados.

Un beneficio adicional sería que las empresas destinen un alto porcentaje a la exportación y no como actualmente que destinan el $77 \%$ de la producción al mercado nacional.

Definitivamente, queda claro que son muy importantes los aportes sociales en las zonas francas por cuanto la generación de empleos, ingresos, los aporte a la salud y a los para fiscales ( hoy CREE), los cuales sumados a los económicos, superan la no despreciable suma \$ 606.101.372.104 millones, en el 2012, y de esa manera en el periodo estudiado, los costos en promedio representaron el $0.058 \%$ de los beneficios totales, siendo el más alto de los cuatro años del estudio, los demás años son insignificantes. 
Cuadro No. 5.

Costos en las Zonas Francas

\begin{tabular}{|c|c|c|c|c|c|c|c|}
\hline \multicolumn{8}{|c|}{ COSTO ZONAS FRANCAS } \\
\hline \multicolumn{8}{|c|}{ VALORES \$ MILLONES } \\
\hline AÑo & 2006 & 2007 & 2008 & 2009 & 2010 & 2011 & 2012 \\
\hline $\begin{array}{l}\text { IMPUESTO RENTA } \\
\text { EXONERADO }\end{array}$ & $(6,405)$ & $(9,576)$ & 111,968 & 92,889 & 81,750 & 134,963 & 160,999 \\
\hline IVA EXONERADO & 11,554 & 15,142 & 627,397 & 588,603 & 661,809 & 887,662 & $3,712,376$ \\
\hline BALANZA \$ MILLONES & (279) & 1,273 & $(3,449,604)$ & $(2,636,674)$ & $(2,145,906)$ & $(1,964,229)$ & $(3,140,667)$ \\
\hline IMPUESTO ARANCEL & 10,511 & 53,730 & $151,200,053$ & $128,078,761$ & $213,360,942$ & $280,144,860$ & $345,174,327$ \\
\hline TOTAL COSTO & 22,064 & 67,598 & $155,389,022$ & $126,125,588$ & $216,250,407$ & $283,131,714$ & $352,188,069$ \\
\hline
\end{tabular}

Fuente: Elaboración propia con datos Banco de la República.

Cuadro No. 6.

Beneficios y Costos

\begin{tabular}{|l|c|c|c|c|c|c|c|}
\hline \multicolumn{7}{|c|}{ Valores en \$ millones } \\
\hline INDICADOR & 2006 & 2007 & 2008 & 2009 & 2010 & 2011 & 2012 \\
\hline $\begin{array}{l}\text { BENEFICIOS } \\
\text { ECONÓMICOS }\end{array}$ & $109,258,655$ & $247,206,010$ & $246,653,931$ & $115,799,834$ & $277,973,846$ & $330,341,246$ & $213,272,104$ \\
\hline BENEFICIOSOCIAL & $1,344,050,000$ & $3,070,116,000$ & $359,250,164,000$ & $531,809,172,000$ & $557,005,762,010$ & $605,888,100,000$ & $605,888,100,000$ \\
\hline BENEFICIOTOTAL & $1,453,318,655$ & $3,317,322,010$ & $359,505,817,931$ & $531,924,971,834$ & $557,284,735,856$ & $605,218,441,245$ & $605,101,372,104$ \\
\hline COSTOS & 28,749 & 79,721 & $155,389,022$ & $123,396,927$ & $216,250,407$ & $283,131,714$ & $352,188,069$ \\
\hline $\begin{array}{l}\text { \% COSTO } \\
\text { IBENEFICIOTOTAL }\end{array}$ & $0.002 \%$ & $0.002 \%$ & $0.043 \%$ & $0.023 \%$ & $0.039 \%$ & $0.047 \%$ & $0.058 \%$ \\
\hline $\begin{array}{l}\text { \% } \\
\text { COSTO/BENENEFIC } \\
\text { IO ECONÓMICO }\end{array}$ & $0.03 \%$ & $0.03 \%$ & $63.00 \%$ & $106.56 \%$ & $77.80 \%$ & $85.71 \%$ & $165.14 \%$ \\
\hline $\begin{array}{l}\text { \% COSTO } \\
\text { IBENEFICIOSOCIAL }\end{array}$ & $0.0021 \%$ & $0.0026 \%$ & $0.0433 \%$ & $0.0232 \%$ & $0.0388 \%$ & $0.0467 \%$ & $0.0581 \%$ \\
\hline
\end{tabular}

Fuente: Elaboración propia con datos Banco de la República.

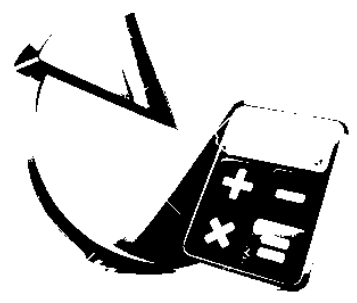

Las empresas de la zona franca y la generación de valor

Una de las mayores preocupaciones de los inversionistas en las empresas es la rentabilidad que pueda reportar los recursos aplicados a las sociedades y especialmente cuál es el valor que crea ésta organización en el tiempo.

Aumentar el valor de la empresa y, por lo tanto, rentabilidad para los propietarios, así como beneficios para sus stakeholders o grupos de interés como empleados, directivos, accionistas, clientes, proveedores, aliados 
Enrique Pinzón Álvarez - Julio Enrique Lora Suarez

estratégicos, entre otros; es una forma de medir el crecimiento del negocio. Hoy en día no es suficiente obtener una utilidad neta positiva ni unos indicadores de medición aceptables, también es necesario contar con una herramienta que permita evaluar el valor del negocio, para ello se puede utilizar el (EVA®) Valor Económico Agregado. Una compañía sólo crea valor cuando es capaz de lograr inversiones que renten más que el costo del capital invertido. El primer paso es considerar la maximización de valor en el largo plazo como uno de los objetivos estratégicos financieros, que permita garantizar la duración de la empresa. Una de las mayores virtudes del EVA® consiste en ayudar a identificar las áreas de la administración donde se crea y se destruye valor. En el país la idea de que las empresas se ubiquen en la zona franca, además de verse beneficiadas con los descuentos tributarios, también se espera que estas ventajas faciliten la generación de valor.

Gráfica No. 6.

Zona franca industria por sectores valor agregado mercado MVA® 2006-2012
El Valor de mercado agregado MVA® es el comportamiento del EVA® en el tiempo, descontado su valor presente y permite ver el valor de mercado agregado o sustraído del capital. A diferencia de la tasa de retorno, la cual refleja el resultado de un período, el MVA® es una medida acumulativa del desempeño corporativo, el cual refleja qué tan exitosamente la empresa ha invertido el capital en el pasado y qué tan bien lo hará en el futuro. (University of Pittsburgh, 2008). Para el análisis de las empresas en zonas francas tomaremos información desde que están reportados en la zonas francas, en algunos casos 7 años, otros sólo 2 años. En el área Industrial se dividió en 19 subsectores, de acuerdo con la información financiera que reportaron las empresas, en algunos sectores, el EVA® muestra un valor negativo muy alto, analizando el comportamiento por compañía se encontró que mínimo una empresa de cada sector presenta un valor muy negativo, razón por la cual se eliminó dicha empresa del análisis. Con dicha eliminación se da un cambio importante, hasta el punto de pasar a ser el EVA® del sector positivo, como se puede observar en la (Gráfica No. $6)$.

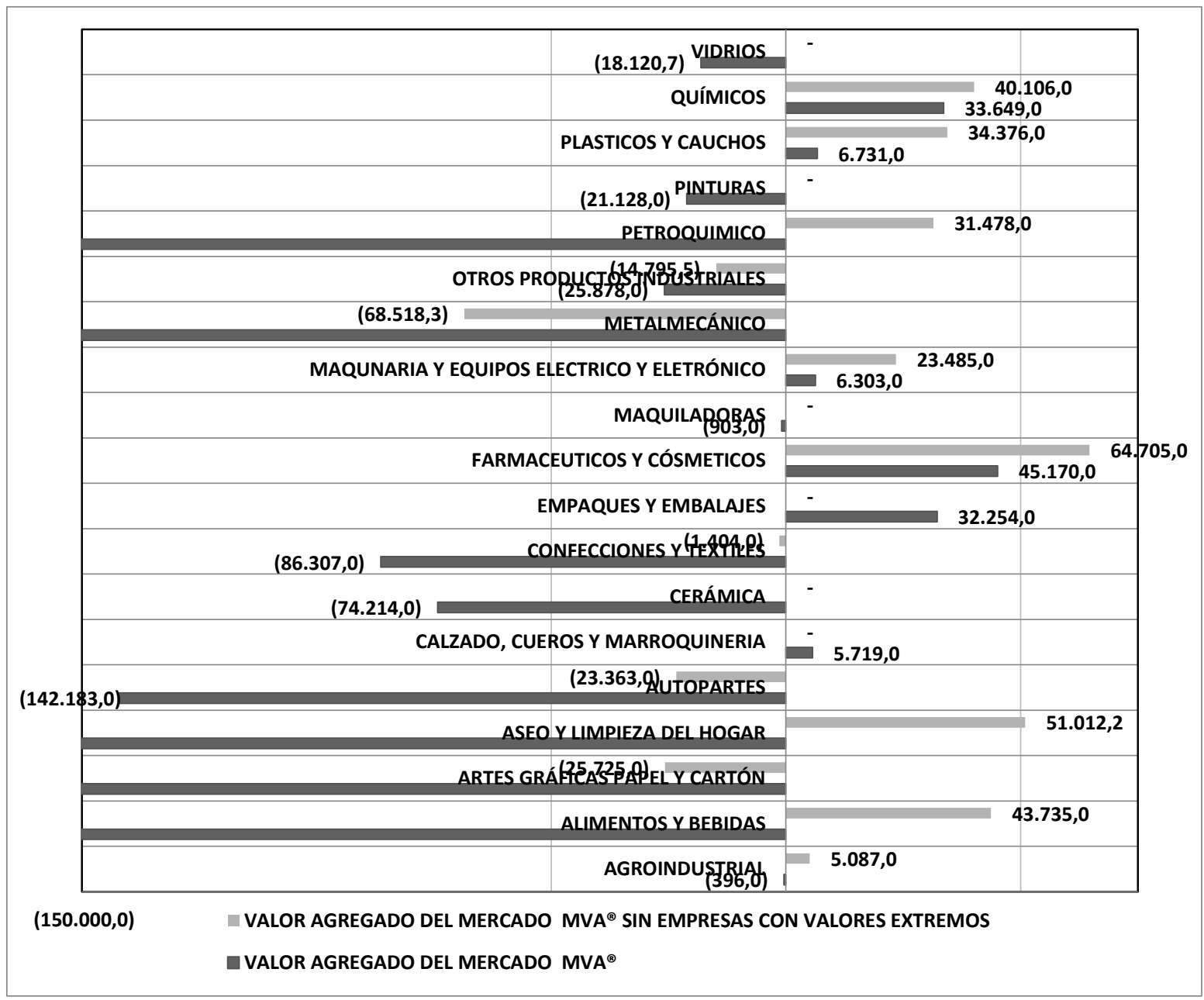


ISSN: 1794-9920 Julio - Diciembre 2015

En la gráfica encontramos que 5 de los subsectores, seguían perdiendo valor aún eliminando la empresa con el mayor valor del mercado agregado MVA® negativo. Estos subsectores son el metalmecánico con $\$ 68.518$ millones, artes gráficas papel y cartón con $\$ 25.725$, autopartes con $\$ 23.323$, productos industriales 14.795 y confecciones y

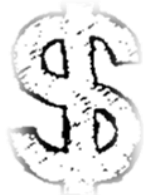
textiles con $\$ 1.404$ millones.

Gráfica No. 7.

Zona franca servicios por sectores valor agregado mercado MVA®

2006-2012

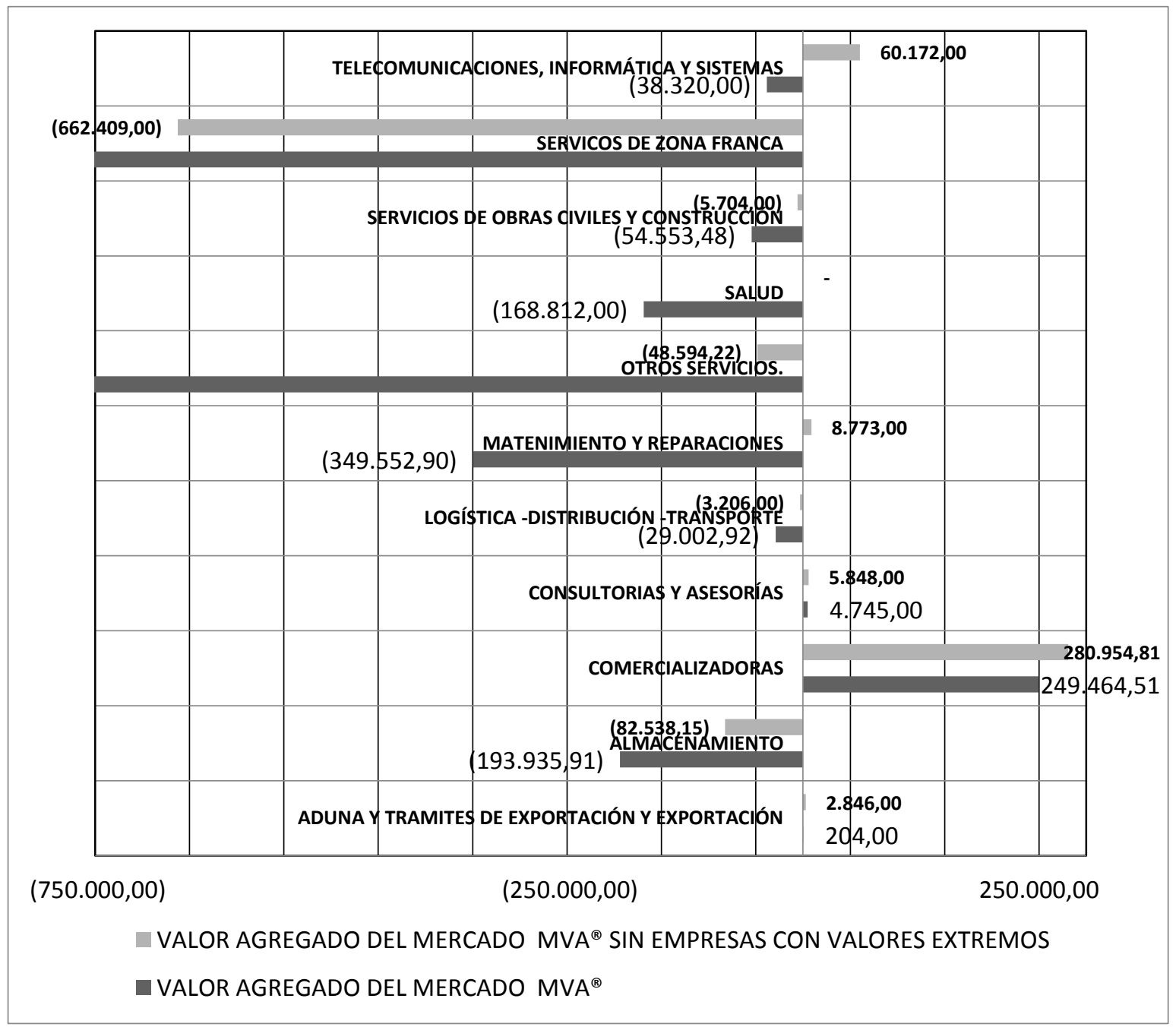

Fuente: Elaboración propia con datos Banco de la República 


\section{LA GENERACIÓN DE BENEFICIOS SOCIALES Y ECONÓMICOS DE LAS ZONAS FRANCAS EN COLOMBIA}

Enrique Pinzón Álvarez - Julio Enrique Lora Suarez

En total de las empresas de la industria, se presentan en promedio un valor agregado de $\$ 8.430$ millones, siendo importante para el país y los inversionistas. Se puede concluir que en 8 subsectores de las zonas francas, para el período 2006-2012, han generado algún valor, 6 subsectores no han generado valor y en 5 subsectores se está destruyendo valor. En el VALOR DEL MERCADO AGREGADO MVA® del sector de servicios (Gráfica No. 7) encontramos que 5 de los sub sectores una vez eliminada la empresa por sector cuya MVA® presentaba el mayor valor negativo, seguían perdiendo valor, estos son el de servicios de zonas francas con $\$ 662.409$, almacenamiento con $\$ 82.538$ y otros servicios con $\$ 48.594$ millones.

Los subsectores que presentan saldos positivos, encontramos las comercializadoras con $\$ 280.955$ millones, seguidos en una proporción inferior por mantenimiento y reparaciones con $\$ 8.773$ y subsector de aduanas y trámites de importaciones y exportaciones con \$2.846. Las empresas de servicios eliminando los MVA® de las empresas más negativos por cada sector, presentan en promedio un saldo negativo de $\$ 40.350$ millones, deduciéndose que no es un negocio rentable.

Se podría decir que 5 subsectores tienen empresas en las zonas francas que en el periodo 2006-2012 han generado algún valor, un subsector no ha generado valor y 5 subsectores están destruyendo valor.

\section{CONCLUSIONES:}

Los beneficios económicos y sociales son significativos en términos absolutos, en el 2012 con la no despreciable suma de $\$ 606,101,372,104$ millones, los costos totales para el estado, en promedio en seis años representan el $1 \%$.

Los beneficios económicos frente a los costos muestran para los siete años, que en promedio por cada peso generado al estado su costo fue del $80 \%$ (\$0.80), que es muy elevado.

En la inversión hay que diferenciar los compromisos de las zonas francas y las inversiones de los usuarios. El monto de inversión de las zonas francas proyectado para el 2013 según el Ministerio de Comercio Industria y Comercio, era de $\$ 7.358$ millones mientras que los usuarios al 2012 invirtieron $\$ 83.622 .155$ millones.

En materia de producción el mayor aporte están el sector metalmecánico, alimentos y bebidas, confecciones y textiles, petroquímica, artes gráficas papeles y cartones, servicios de salud, mantenimiento y obras civiles.

El empleo directo generado hasta el 2012 ascendía a 42.825 personas ocupadas. Los sectores que más cargos generaron son el de textiles y confecciones $12.71 \%$ (5.443), empresas de comercialización 11.98\% (5.130).

En materia de comercio exterior se observa un déficit permanente en las exportaciones, generando una balanza deficitaria de US $\$ 1.750$ millones en el 2012, en contraste con la balanza comercial del país, que obtuvo un superávit de US\$56.953.500. En la medida que entren plenamente a producir las empresas recién instaladas en su capacidad máxima, los costos para el estado tienden a ser inferiores.

El impuesto a la renta tiene una tenencia ascendente a partir del 2006, el exonerado en el 2012 representó el 31\% frente al pagado que fue del $69 \%$. El comportamiento del impuesto de valor agregado es similar al de renta y su crecimiento en los siete años es del $321 \%$, por la razón ya comentadas de la producción con destino al mercado doméstico.

Las inversiones de los empresarios en las zonas francas en promedio de los 7 años del estudio alcanzaron $\$ 20$ mil millones, superando los compromisos de inversión exigidos por el gobierno que para el 2013 eran en total \$ 14 mil millones.

Este artículo permitió demostrar que en los beneficios económicos sobresalen producción, el capital de las empresas incorporadas a zonas francas, impuestos de valor agregado (IVA).

Finalmente la generación de valor por las empresas de las zonas francas tanto en los sectores de la industria y servicios se ve más afectada porque las empresas cuentan con un costo financiero alto, a pesar de los beneficios tributarios que estimulan a los empresarios a ubicarse en dichas áreas.

\section{REFERENCIAS:}

Departamento Administrativo Nacional de Estadítica DANE, (2013). Estadisticas Comercio Internacional. Recuperado 10 junio 2013 de Dane: http://www.dane.gov.co/index.php/estadisticaspor-tema/comercio-internacional 
EVA® for Small Manufacturing Companies, (2008). University of Pittsburgh, Department of Industrial Engineering.

Granados, J. (2003). Zonas francas y otros régimenes especiales en un contexto de negociaciones multilaterales y regionales. Instituto para la integración de america latina y del caribe.Chile.

Gomez, H \& Leon, A. (2010). Institucionalidad estrategia para el desarrollo exportador y la diagnostico inicial e innovación en Colombia, Comision economica para america latina y el caribe Cepal. Chile.

Ibarra, MG. (2012). Papel de las zonas francas en la integracion de los procesos productivos entre estados unidos canada colombia y america latina. congreso zonas francas. Colombia. Consulta 1 junio 2013.

Mongue, R. (2005). Análisis costo beneficio del régimen de zonas francas, impacto de la inversión extranjera directa en costa rica. Organización de los estados americanos. Washington (U.S.A.)

Organización Mundial de Comercio OMC. (2013). Informe sobre el comercio mundial. Recuperado 10 de Mayo 2013, de OMC13:

https://www.wto.org/spanish/res_s/booksp_s/anal ytic index s/agriculture 02 s.htm

Pinzón, E. (2013). Las zonas francas con beneficios tributarios genera productos para el mercado nacional y no para la exportacion. Revista Análisis Internacional RAI, 8, 147-163.

Torres, R (2008). Análisis de la política comercial y su impacto en las exportaciones industriales colombianas (1990-2011). Universidad Jorge Tadeo Lozano de Bogotá, Colombia. 